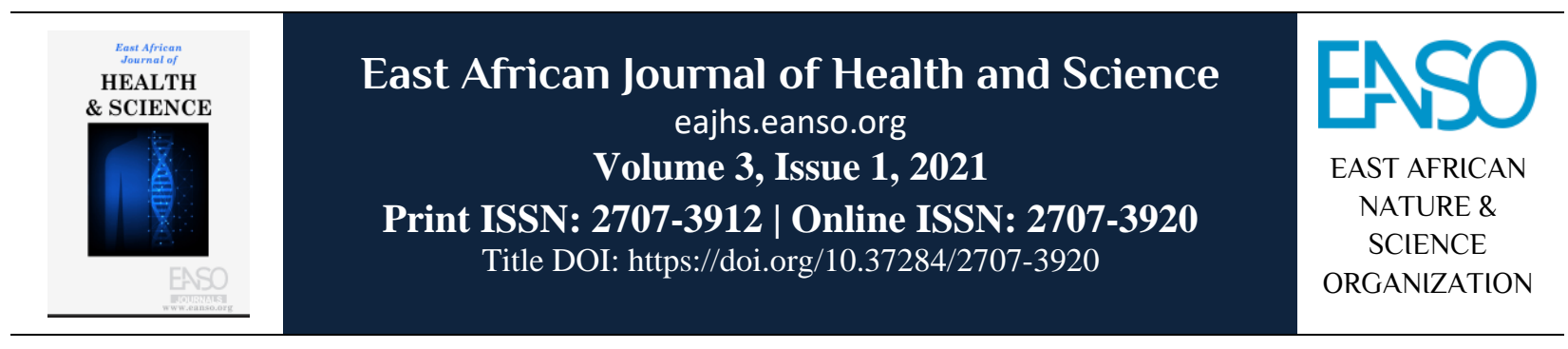

Original Article

\title{
Respectful Maternity Care Interventions: A Systematic Literature Review.
}

\author{
Lucy Natecho Namusonge ${ }^{1 *} \&$ Jacob Odhiambo Ngachra ${ }^{2}$ \\ ${ }^{1}$ Kibabii University, P. O. Box 2008 - 50200, Bungoma, Kenya. \\ ${ }^{2}$ Muhoroni County Hospital, P. O. Box 486 - 40100, Kisumu, Kenya. \\ *Author for correspondence email: jacoodhiambo78@gmail.com.
}

Article DOI: https://doi.org/10.37284/eajhs.3.1.330

\section{Date Published: ABSTRACT}

20 May 2021 High maternal and new born mortality remains a pressing problem in developing settings. Poor treatment during childbirth contributes directly and indirectly to this

Keywords: problem at a rate of $82 \%$. Many women experience disrespectful and abusive treatment during childbirth in facilities worldwide which violates their rights to

Autonomy, respectful care and a threat to their right to life, health, bodily integrity and freedom

Health, from discrimination. Majority of women report to have experienced some form of Maternity Care, Mortality Ratio, Maternal Health, New Born Care, New Born Health. disrespect and abuse during childbirth. This systematic literature review aimed at reviewing studies on Respectful Maternity Care interventions aimed at promoting Quality of Maternal and New born Care. Objectives were to assess literature on how mothers are treated during childbirth (experience of care/respectful maternity care), to find out the factors contributing to disrespect and abuse during childbirth, to identify strategies for addressing issues affecting respectful maternity care. The literature review employed a quality assessment tool 'PRISMA', by the WHO. Eligibility criteria was studies published between 1992 and 2020 in indexed national and international journals in English language focusing on Respectful Maternity Care interventions in promoting Quality of Maternal and New born Care. Literature was reviewed from academic databases, project reports and documents. Electronic searches included reference libraries: PubMed, Cochrane Reference Libraries, google scholar, Medline on Mega scope data bases, CINAHL, Embase data bases and grey literature. Studies meeting the following criteria were included in the review: 1) Respectful Maternity Care; 2) Facilitators to dignity and respect during childbirth; and 3) Quality of Maternal and New born Care (QMNC). Disrespect and abuse appear to be widespread and research and implementation efforts must continue, there is lack of standardized definitions, instruments hence affecting generalizability and comparability, there are no adequate available data

45 | This work is licensed under a Creative Commons Attribution 4.0 International License. 
to quantify the prevalence of these behaviours. No matter the conceptual framework used in future investigations, studies should seek to accomplish three objectives: (1) to measure the perceived and observed frequencies of mistreatment in maternal health settings, (2) to examine the macro and micro level factors that drive mistreatment, (3) to assess the impact of mistreatment on the health outcomes of women and their new borns, and 4) to employ mixed method design as an optimal strategy to evaluate mistreatment and include direct observations to bridge the gap between observed measures and participants' self-reported experiences of mistreatment.

\section{APA CITATION}

Namusonge, L. N., \& Ngachra, J. O. (2021). Respectful Maternity Care Interventions: A Systematic Literature Review. East African Journal of Health and Science, 3(1), 45-58. https://doi.org/10.37284/eajhs.3.1. 330.

\section{CHICAGO CITATION}

Namusonge, Lucy Natecho., \& Jacob Odhiambo Ngachra. 2021. "Respectful Maternity Care Interventions: A Systematic Literature Review.”. East African Journal of Health and Science 3 (1), 45-58. https://doi.org/10.37284/eajhs.3.1. 330.

\section{HARVARD CITATION}

Namusonge, L. N., \& Ngachra J. O. (2021) "Respectful Maternity Care Interventions: A Systematic Literature Review", East African Journal of Health and Science, 3(1), pp. 45-58. doi: 10.37284/eajhs.3.1. 330.

\section{IEEE CITATION}

L. N. Namusonge and J. O. Ngachra, "Respectful Maternity Care Interventions: A Systematic Literature Review", EAJHS, vol. 3, no. 1, pp. 45-58, May. 2021.

\section{MLA CITATION}

Namusonge, Lucy Natecho., \& Jacob Odhiambo Ngachra. "Respectful Maternity Care Interventions: A Systematic Literature Review". East African Journal of Health and Science, Vol. 3, no. 1, May 2021, pp. 45-58, doi:10.37284/eajhs.3.1.330.

\section{INTRODUCTION}

High maternal and newborn mortality and morbidity remains a pressing problem in developing settings (Furuta et al., 2011). Poor treatment during childbirth contributes both directly and indirectly to this problem. Treatment refers to care during childbirth that is respectful and responsive to individual women and their families' preferences, needs, and values. It emphasizes the quality of patient experience (Afulani et al., 2017). The evidences based on safety, its root causes and contributing factors, as well as the cost-effective solutions to common problems are precisely limited (Esmail et al., 2017). Due to this fact, research on maternal and neonatal care has been acknowledged by the World Health Organization (WHO) Patient Safety Programme as one of the top 20 global research priorities in low-income countries whose economies are in transition (World Health Organization, 2010).

The United Nations Sustainable Development Goals (SDGs) have set ambitious health-related targets for mothers, newborns and children, which countries have devoted to achieve by 2030 . Working towards these means progress is made on universal health coverage (UHC) and on attaining the goals of the Global Strategy for Women's, Children's and Adolescents' Health as well as the goals of Every Woman Every Child movement (World Health Organization, 2018). According to WHO's document on trends in maternal and newborn mortality, the goal is to fast-track the lessening of maternal and newborn morbidity and mortality towards the achievement of the SDGs by safeguarding healthy lives and promoting wellbeing at all levels including global reduction of MMR to less than 70/100,000 live births and reduction of neonatal mortality to at least as low as

46 | This work is licensed under a Creative Commons Attribution 4.0 International License. 
12 per 1000 live births by 2030 (World Health Organization, 2014).

Maternal and neonatal morbidity and mortality rates continue to be recognized internationally as public health priorities. Moreover, these indicators in Africa have continued to rise instead of declining since launch of Safe Motherhood Initiative 31 years ago (Dickson et al., 2015). It is shown that $99 \%$ of all maternal and neonatal deaths occur in low and middle-income countries with Sub-Saharan Africa accounting for almost $66 \%$ of global maternal mortalities. It has the utmost maternal mortality ratio of 900 maternal deaths per 100,000 live births and also the highest lifetime risk of maternal death of 1:26. Neonatal deaths are estimated at 27 per 1000 live births [NCK, 2012; National Road Map, 2010).

The rate of skilled birth attendance uptake has gone up from $58 \%$ in 1990 to $73 \%$ in 2013 , mainly due to increases in facility-based births. Evidence shows, however, that giving birth in a health facility with a skilled attendant is not sufficient to reduce maternal and newborn deaths and severe morbidity. Many women and their babies die as a result of poor care, even after reaching a health facility (Bohren et al., 2014). Accessing labour and childbirth care in health care facilities may not guarantee good quality care. Disrespectful and undignified care is prevalent in many facility settings globally, particularly for underprivileged populations, and this not only violates their human rights but is also a significant barrier to accessing intrapartum care services. Despite there being many anecdotal reports on Disrespect and Abuse, there is little formal research done and no normative standard for respectful care (Bohren et al., 2015).

There is dire need to care for women with complications of pregnancy, childbirth or the immediate postpartum period, including immediate problems of the newborn (Turab et al., 2013). 60\% of deaths are attributed to lack of access to skilled birth attendance by pregnant women and inadequate knowledge and skills of healthcare providers in emergency obstetrics and neonatal care as well as mistreatment of women during childbirth that leads to mismanagement (Turab et al., 2013).
Timing is critical in preventing maternal and newborn mortality and morbidity. Post-Partum Haemorrhage (PPH) can kill a woman in less than 2 hours; for antepartum haemorrhage, eclampsia, obstructed labour, and sepsis, the times they must receive emergency care would be 12 hours, 2 days, 3 days, and 6 days, respectively. Most perinatal deaths occur during labour and delivery or during the following 48 hours. A majority of the maternal and early newborn deaths can be avoided by ensuring prompt and respectful care (Gabrysch \& Campbell, 2015). According to WHO, every woman has the right to the highest attainable standard of health, which includes, the right to dignified, respectful healthcare (World Health Organization, 2014).

Maternity care is related to pregnancy, childbirth, and the postpartum period aimed at improving maternal and newborn health outcomes. It includes monitoring the wealth and wellbeing of the mother and baby, health education, and assistance during childbirth. Its components entail Respectful Maternity Care (RMC); Emergency Obstetric and Newborn Care (EmONC); Essential Newborn Care (ENC); Focused Antenatal Care (FANC); and Malaria in Pregnancy (MIP) (Wiegers T.A. 2009). This study focused on Respectful Maternity care in promoting quality as a component of maternity care.

In 2009, the Health Research Program initiated research to generate evidence and focus global attention on disrespect and abuse during facilitybased childbirth. These efforts led to a global movement to promote Respectful Maternity Care (RMC) as a human right. Prior to 2009, there was limited documentation of the scope and impact of disrespect and abuse in health facilities during childbirth (World Health Organization, 2014). Respectful maternity care advocates suggest that safe motherhood must be expanded beyond the prevention of illness or death to include respect for women's basic human rights including respect for women's autonomy, dignity, feelings, choices and preferences (White Ribbon Alliance, 2011).

Currently, Respectful Maternity Care is a top priority in the World Health Organization (WHO) recommendations on intrapartum care or a positive childbirth experience. The WHO recommends provision of respectful maternity care in accordance

47 This work is licensed under a Creative Commons Attribution 4.0 International License. 
with the human rights-based approach to decrease maternal and newborn morbidity and mortality and improve women's experience of labour and childbirth and address health disparities (World Health Organization, 2018). WHO identified RMC as a key component of quality care in the WHO Quality of MNH Care Frame work. RMC continues to gain prominence around the world. Afghanistan, India, Kenya, Malawi, Nepal, and Nigeria integrated RMC into national policy, standards of maternal care, and training of providers (Ramsey et al., 2016).

No woman should be hit, yelled at, or abused in any way during childbirth. However, labouring women in Kenya and elsewhere may experience inhumane treatment at hospitals and clinics. This abuse is key yet overlooked reason that 4 in 10 pregnant women in Kenya deliver at health facilities (Kenya National Bureau of Statistics, 2015). A study carried out in Kakamega County on women's persistent utilization of unskilled birth attendance showed that health providers' negative attitude contributed $43.6 \%$ to this problem (Namusonge et al., 2016). Promoting respect and dignity is a key component in providing quality care during facility-based childbirth and is becoming a critical indicator of maternal healthcare. Providing quality care requires essential skills and attitudes from healthcare providers, as their role is central to optimizing interventions in maternity settings (Ndwiga, 2017).

\section{MATERIALS AND METHODS}

A quality assessment tool 'PRISMA', by the World Health Organization (WHO) was adopted to perform the literature review (Moher et al., 2009).

\section{Eligibility}

Studies published between 1992 and 2020 in indexed national and international journals in the English language focusing on Respectful Maternity Care interventions in promoting Quality of Maternal and Newburn Care (QMNC). Respectful Maternity Care (RMC) refers to care organized for and provided to all childbearing women in a manner that maintains their humanity, dignity, privacy and confidentiality, ensures freedom from harm and mistreatment, and enables informed choice and continuous support during labour and childbirth (Bowser \& Hill, 2010).

\section{Identification of Studies}

Literature was reviewed from academic databases, as well as project reports and documents. Key sources of electronic searches included reference libraries, namely: PubMed, Cochrane Reference Libraries, google scholar, Medline on Mega scope data bases, CINAHL, Embase data bases grey literature was searched using predetermined search strategy to identify qualitative, quantitative, and mixed method studies on respectful maternity care across all geographical and income-level settings. The goal was to access data on Respectful Maternity Care (RMC) interventions in promoting Quality of Maternal and Newborn Care (QMNC). While searching the databases, various medical subject key words were used such as 'maternal health', 'quality care', 'newborn care', 'respectful maternity care', 'newborn health', 'strategies', 'effectiveness' and 'evaluation'.

\section{Study Selection}

All the study titles and abstracts were initially screened and the process repeated to ensure consistency. Studies which met one of the following criteria were included in the review: 1) Respectful Maternity Care (Treatment of mothers/experience of care during childbirth); 2. Facilitators to respect and dignity during childbirth; and 3) Quality of Maternal and Newburn Care (QMNC). Moreover, only those selected studies as per the criteria were reviewed, where respectful maternity care Quality Improvement (QI) approaches/interventions had the potential of scaling up.

\section{Data Extraction}

Data was extracted from all the studies found to be fulfilling the above criteria. Two researchers independently reviewed the full texts of these papers. A spread sheet was used for data extraction. Finally, both researchers critically reviewed all the selected studies and resolved any observed inconsistencies through discussion. The data was extracted using the following parameters: 1) geographical setting and target population; 2) description of Respectful Maternity Care

48 This work is licensed under a Creative Commons Attribution 4.0 International License. 
approaches/interventions and relevant set of services provided; 3) outcome; and 4) key findings highlighting Quality of Maternal and New born Care

\section{Synthesis of Results}

Analytic approach of thematic analysis was used, in which data from selected studies were categorized, grouped and interpreted according to the prominent themes with the aim of identifying common elements across the studies (Austin et al., 2014). The analysis began with a deductive approach through which emerging themes and sub-themes were initially identified. All Respectful Maternity Care approaches/interventions implemented to enhance Quality of Maternal and New born Care, were analysed considering their quality measures, the strategies adopted, the main results and outcomes. Additionally, they were grouped together to highlight key themes and summarized in tabular form.

\section{RESULTS}

The electronic data base yielded a large number of studies, which reflected the widespread discussion on Respectful Maternity Care approaches/interventions in promoting Quality of Maternal and New born Care. A total of 187 unique records were retrieved out of which 129 full texts were screened. Records identified from other sources were 10. Finally, 60 studies on Respectful Maternity Care interventions/approaches were selected for analysis. In Table 1, the flow of studies through searching and screening for inclusion is described.

\section{Figure 1: PRISMA flowchart for study selection}

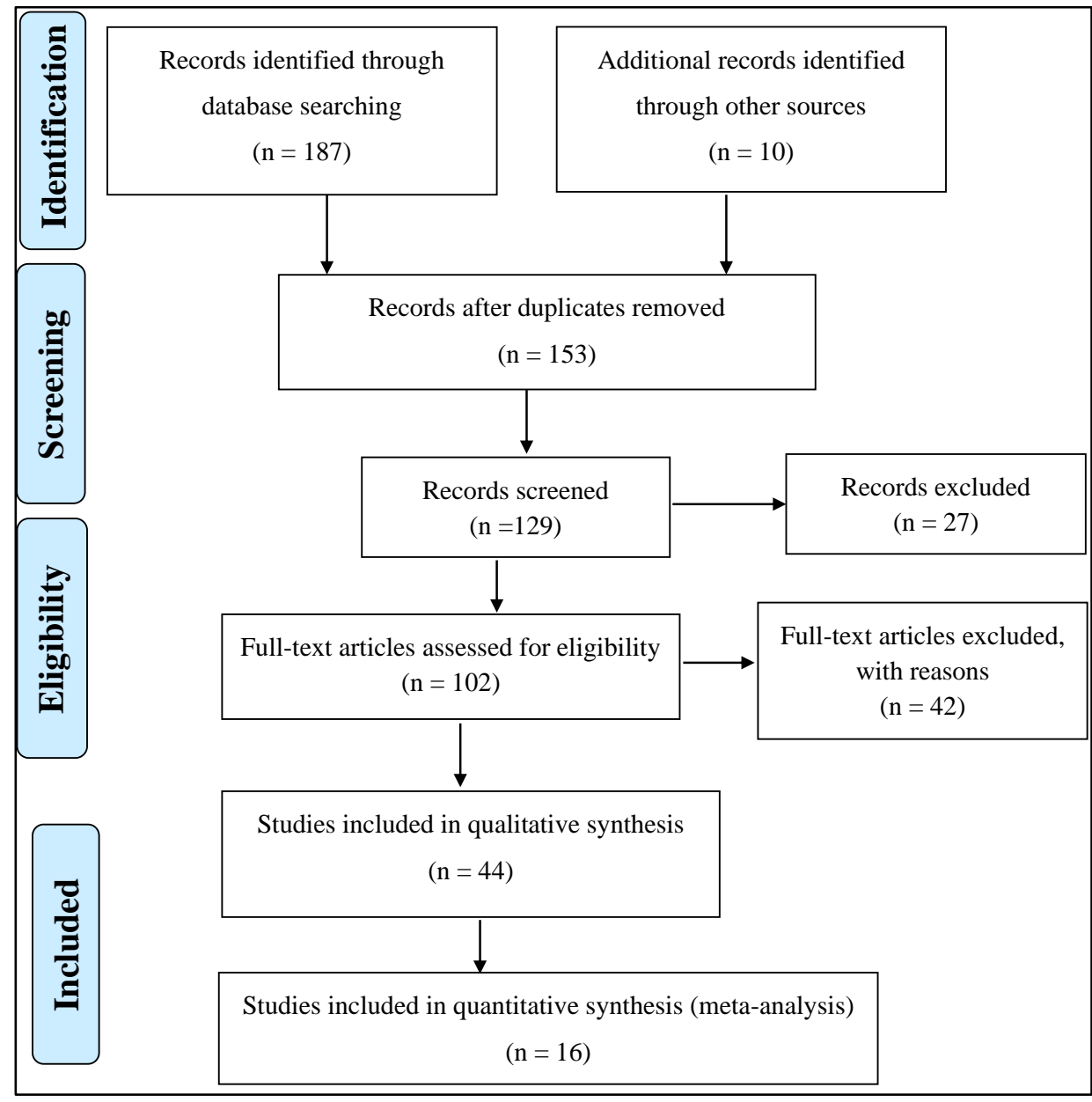

49 | This work is licensed under a Creative Commons Attribution 4.0 International License. 


\section{Respectful Maternity Care Quality Improvement Approaches/Interventions}

The analysis of selected studies highlighted three broad cross cutting issues as the commonly applied Respectful Maternity Care interventions/approaches for improving Quality of Maternal and New born Care. Thirteen (13) studies focused on capacity building of health workers on Respectful Maternity Care and clinical quality, nine (9) focused on clinical audits and feedback, three (3) studies focused on improvement of health workers performance, and supportive supervision, three (3) studies focused on community engagement, and thirty-two (32) studies focused on multidimensional interventions.

\section{Capacity Building of Health Workers on Respectful Maternity Care and Clinical Quality}

Capacity building of healthcare providers on improvement of quality, knowledge levels and adherence to protocols is a proven approach for enhancing Respectful Maternity Care. It was found out that moderate-certainty evidence suggested that women are more likely to report experiencing respectful care with RMC interventions/training than without RMC interventions/training. It's also concluded that women placed high value on RMC, and providers perceived RMC to be a critical component of providing safe and good quality (World Health Organisation, 2018). A study was carried out in the Philippines to question the reasons behind disrespect and abuse and it was concluded that the situation was complicated. Most clinicians did not go to work seeking to shame and neglect women. Healthcare providers are expected to work in poor conditions that exacerbate this behaviour. There are three deadly delays a woman can face during labour: not recognizing the signs of an emergency and seeking care; not identifying and reaching a medical facility; and not receiving adequate and appropriate treatment. Disrespect and abuse contribute to delays \#1 and \#2. If a woman does not feel safe and respected when she first visits a maternity centre, she is less likely to attend her antenatal care appointments or to come to the facility when in labour her risk of both pregnancy related morbidity and mortality. And if she does make it to the facility for delivery but receives poor care, she and her child remain at risk (Bartlett, 2015).

Kenya is among countries honoured by WHO for its contributions to enhancing RMC. Evidence suggests that women who give birth in health facilities can face humiliating and undignified conditions- from physical abuse, non-confidential care, non-dignified care, abandonment and discrimination, to unfair requests for payment. Provision and experience of care- how women are treated-are both critical components of quality that can influence service utilization and outcomes (Ndwiga et al., 2017; World Health Organization, 2018). Care navigators are specifically chosen and trained to gain the trust and respect of patients, traditional midwives and biomedical providers- and to decrease opposition to hospital level obstetric care hence overcoming "humanistic barrier" (Afulani et al., 2017; Austad et al., 2018).

A tool was developed on patient-centred maternity care (PCMC) which is recognized as critical to improving reproductive health outcomes, yet little research exists on how to operationalize it. Future studies can validate the tool to assess its appropriateness for the setting it is to be used. It can be administered as a quantitative measurement through exit interviews, community interviews as well as through before and after interventions to improve women's experience during childbirth- for needs assessment as well as for monitoring and evaluation of interventions (Afulani et al., 2017; Nababan et al., 2017).

The predominant model of childbirth care is characterized by the abusive or inappropriate use of interventions (routine amniotomy, lithotomy and intravenous infusion of oxytocin and restriction on the presence of a birth companion in all stages of labour. Researchers recommended that there should be some changes in labour and delivery care, including the need to rescue the idea of childbirth as a natural event, with a stimulus for the work of the midwives, the use of practices based on the best scientific evidence and access to appropriate technologies for childbirth care (Cortes et al., 2018). A study was carried out in 44 public health facilities in Rajasthan, India with the aim of improving the quality of childbirth services through orientation training of doctors and program managers and

50 This work is licensed under a Creative Commons Attribution 4.0 International License. 
regular visits to facilities involvement assessment, feedback, training and action. Outcome evaluation showed a significant reduction in unnecessary or harmful practices such as fundal pressure (Iyengar et al., 2014). A descriptive, cross-sectional quantitative study was undertaken in Brazil at the Assis Chateubriand Maternity Hospital with a sample of 62 companions through interviews and a survey. Findings showed that majority of the companions who witnessed birth did not undergo any training, presence of a birth companion was considered to have positive outcomes (Oliveira et al., 2014).

If we want to accelerate reductions in maternal and newborn morbidity and mortality, improvement the quality of care and patient safety is critical. WHO envisions a world in which "every pregnant woman and newborn receives quality care throughout pregnancy, childbirth and the postnatal period" (World Health Statistics, 2016). The burden of maternal and perinatal deaths is disproportionately higher in low-and middle-income countries (LMICs) compared to high-income countries (HICs). Therefore, improving the quality of care especially in LMICs has been identified as the most impactful strategy for reducing stillbirths, maternal and newborn deaths (Bohren et al., 2015). Deaths within the first few hours after childbirth contribute to a significant proportion of maternal and neonatal mortality in developing countries. Skilled birth attendance and timely action to detect and address complications are essential for reducing maternal and perinatal mortality. To impact mortality however, the rising rate of institutional delivery must be accompanied by commensurate improvements in quality of care (Hulton et al., 2000; Iyengar et al., 2014).

Access to and availability of medical care are both necessary but not sufficient factors to improve maternal and newborn health, in fact, they do not guarantee increased utilization of services and improved client satisfaction. Poor quality of care is the most plausible explanation for this reality. There should be improvement in the health system's capacity to provide quality care (Souza et al., 2013; Van den Broek \& Graham, 2009). A growing body of research on women's experiences during pregnancy, and particularly childbirth paints a disturbing picture. Many women across the globe experience disrespectful, abusive or neglectful treatment during childbirth in facilities which can also be a powerful disincentive for women to seek and use healthcare services. Such practices may have direct consequences for both the mother and infant (Furuta et al., 2014; World Health Organization, 2015).

Majority of maternal and newborn deaths in Sub Saharan Africa are associated with birth complications related to lack of trained supervision at delivery, with only $10 \%$ of maternal and newborn deaths attributed to infections or disease (Gabrysch et al., 2012).

\section{Clinical Audits and Feedback}

A baseline survey was carried out in Kenyan 13 health facilities and revealed several facts that emphasize that disrespect and abuse is a pressing problem: 1 out of 5 postpartum women leaving the postnatal ward reported feeling humiliated at some point during their most recent delivery; 9 out of 10 healthcare providers said they had heard of or witnessed colleagues treating women inhumanely. Most facilities had essential equipment and supplies. Younger women and women with higher parity were more likely to be disrespected and abused (Abuya et al., 2015). The usefulness of a midwife has conventionally been associated with maintaining standards of care (Abott et al., 2010).

In a study carried out in Ethiopia on the prevalence of disrespect and abuse experienced by mothers during facility-based childbirth and its associated factors (Siraj et al., 2019). It was found that the prevalence of disrespect and abuse was $91.7 \%$. The most common type of disrespect and abuse were culturally inappropriate care $(75.2 \%)$; failure to encourage the client to ask questions (75.9\%); provider not introducing him/herself (80.0\%); failure to obtain consent/permission prior to any procedure $(63.8 \%)$; and not using curtains/visual barriers to protect client (81.7\%). Efforts ought to be made towards addressing disrespect and abuse in order to improve the quality of maternal and newborn care (Siraj et al., 2019).

A survey was carried out among 653 new mothers in Tshwane Health District, South Africa aimed at determining their experience during childbirth with

51 This work is licensed under a Creative Commons Attribution 4.0 International License. 
a view of improving respectful care practices in low-risk, midwife-led obstetric units. An ANCOVA was performed to identify the sociodemographic variables that significantly predicted disrespectful care. It was found that age, education level and length of residence in the district were significantly associated with disrespectful care. It was concluded that there was need to improving respectful care through interventions that are integrated into routine practices in labour ward. Care should be culturally sensitive and equitable (Oosthuizen et al., 2017). Curl \& Lothian, 2013 found that generational characteristics amongst midwives influenced to some extent the culture of service delivery during childbirth with younger nurses being more abusive and disrespectful. Practice should be supported by top leadership as well as policies and protocols that reflect best evidence and having responsive healthcare systems (Bingham \& Main, 2010; Mwaniki et al., 2014).

\section{Improvement of Health Workers Performance}

Lack of adequate training of healthcare providers may expose apparently healthy pregnant women to unnecessary medical interventions that interfere with their physiological process of childbirth. Health facilities should ensure access to skilled healthcare professionals and timely referrals should the need for additional care arise (Balde et al., 2017; World Health Organization, 2018). An interventional study on respectful maternity care was done which showed a baseline prevalence on disrespect and abuse at $20 \%$ which was lowered to $7 \%$ after sensitization of healthcare providers (Ndwiga, 2017). When providing care to women, obstetric nurses should develop actions geared towards individualized, welcome and efficient care in an environment enabling integral healthcare practices (Silva et al., 2015; Mselle et al., 2018).

\section{Community Engagement}

It is suggested that to advance respectful maternity care for all, we must hear from women themselves about how they define high quality maternal and newborn care and reproductive health services (George-Carey, 2018; Chalmers, 1992). Awareness deficiencies on respectful maternity care should be addressed through maternal and child health services by designing appropriate strategies including provision of targeted information, education and communication as a concurrent measure to quality improvement (Hasting, 2015).

\section{Multidimensional Interventions}

Respectful Maternity Care should be provided to all childbearing women in a manner that maintains their humanity, dignity, privacy and confidentiality, ensures freedom from harm and mistreatment, and enables informed choice and continuous support during labour and childbirth. Poor treatment violates the rights of women to respectful care and also threatens their rights to life, health, bodily integrity and freedom from discrimination (Bowser \& Hill, 2010; World Health Organization \& UNICEF, 2015).

In a study carried out in Kenya by a visiting fellow from Women and Health Initiative, Harvard T.H. Chan School of Public Health, it was found that maternal and neonatal mortalities were caused by lack of funding for maternal health, lack of basic equipment and infrastructure, shortage of human resource, inadequate training and supervision of healthcare providers, negligence, abandonment and unethical practices (George-Carey, 2018). Factors contributing to disrespect and abuse including societal factors and lack of accountability, substandard infrastructure, limited resources, stressful working conditions and poor health worker supervision (Hasting, 2015). Lack of national commitment and financial support, poor coordination amongst spouses, inadequate male involvement together with low status of females with poor decision-making power contributed mistreatment (Mirkuzie et al., 2014).

To enable autonomy and dignity he factors to be considered are promotion of coercion free personal relationships, facilitating access to information, and fostering the active participation of women (Silva et al., 2015; Behruzi et al., 2010). Mistreatment can occur at the level of interaction between the woman and provider, as well as through systemic failures at the health facility and health system levels. A typology needs to be proposed and adopted to describe the phenomena and be used to develop measurement tools and inform research programs and interventions (Bohren et al., 2015).

52 This work is licensed under a Creative Commons Attribution 4.0 International License. 
Methodological approaches on the validity and generalizability of estimates used to measure disrespect and abuse have so far been analysed by one study which found out lack of standardized definitions, instruments, and study methods to quantify disrespect and abuse. By presenting collective lessons learnt about the impact of varying methodological designs on the accuracy and usability of the ensuing prevalence estimates with high validity and generalizability (Sando et al., 2016).

In a systematic literature review done within Latin America and the Caribbean that sought to contribute to the determination of best practices in defining and measuring the mistreatment of women during childbirth, it was found out that for measuring mistreatment, half of the research publications used qualitative methods, such as in-depth interviews and focus groups. After analyzing the strengths and limitations of quantitative, qualitative and mixed methods approaches, the researchers recommended mixed methods designs as the optimal strategy to evaluate mistreatment and advocated the inclusion of direct observations that may help bridge the gap between observed measures and participant's' selfreported experiences of mistreatment. They further recommended that no matter the conceptual framework used in future investigations, future studies should seek to accomplish three objectives: 1) to measure the perceived and observed frequencies of mistreatment in maternal health settings, 2) to examine the macro and micro level factors that drive mistreatment, and 3) to assess the impact of mistreatment on the health outcomes of women and their newborns (Savage \& Castro, 2017; Gagliard et al., 2011).

Women's assessment of quality is central because emotional, cultural and respectful support are vital during labour and delivery process. In Tanzania the women rated quality in terms of adequate medical equipment, health staff, room, water, information, clean environment, privacy and the presence of family members. Cultural inappropriateness of care, disrespectful and inhuman services, and lack of emotional support can deter women from accessing obstetric care (Kruk et al., 2014).

According to the Kenya National Commission on Human Rights, Kenya's public health facilities have long been plagued by reports of abuse, mistreatment, and negligence of patients in the hands-off staff, a problem enhanced by poor supervision and understanding. Patients also report that the public health system is culturally sensitive, failing to adapt to local circumstances such as cultures which requires women to be attended by female practitioners. Health workers are also insufficiently trained (Ndwiga et al., 2017).

A study carried out among childbearing women in Nigeria and Uganda to explore what 'quality of care' means to them as a means of ensuring that women's voices and opinions are prioritized when developing interventions to improve quality in maternity care provision. Effective communication, respect and dignity, emotional support, competent and motivated human resources and essential physical resources were mentioned. Women believed that good quality of care ensured optimal psychological and physiological outcomes for the woman and her baby (Bohren et al., 2017).

Quality improvement interventions should target a multi-dimensional approach. Quality improvement interventions should target infrastructure, equipment and supplies, human resource, monitoring tools. They should also target antenatal, delivery and newborn care services with nurses as the key partners (Das et al., 2018).

\section{CONCLUSION}

This synopsis shows a summary of reviewed literature and gaps.

While there is progress, disrespect and abuse appear to be widespread and research and implementation efforts must continue to ensure that all new mothers receive dignified, positive birth experience they deserve, no matter their circumstances.

The review underscores the need for caution in interpreting or comparing previously reported prevalence estimates of disrespect and abuse (D\&A) during facility-based childbirth. The lack of standardized definitions, instruments, and study methods used to date in studies designed to quantify D\&A in childbirth facilities may introduce systematic error in reported prevalence estimated hence affecting generalizability and comparability. 53 This work is licensed under a Creative Commons Attribution 4.0 International License. 
Despite numerous reports including a wealth of qualitative and legal evidence documenting D\&A of women during facility-based childbirth, until recently there are no adequate available data to quantify the prevalence of these behaviours.

No matter the conceptual framework used in future investigations, studies should seek to accomplish three objectives: (1) to measure the perceived and observed frequencies of mistreatment in maternal health settings, (2) to examine the macro and micro level factors that drive mistreatment, and (3) to assess the impact of mistreatment on the health outcomes of women and their newborns.

More than half of the research publications in this review use qualitative methods, such as in-depth interviews and focus groups. After analysing the strengths and limitations of quantitative, qualitative and mixed methods designs. The researcher recommends mixed method design to be used as an optimal strategy to evaluate mistreatment and advocate for the inclusion of direct observations that may help bridge the gap between observed measures and participants' self-reported experiences of mistreatment.

Disrespect and abuse (D \& A) during labour and delivery are important issues correlated with human rights, equity, and public health that also affect women's decisions to deliver in facilities, which provide appropriate management of maternal and neonatal complications. Little is known about interventions aimed at lowering the frequency of disrespectful and abusive behaviours

\section{RECOMMENDATIONS}

In view of the identified gaps in previous studies, future studies should endeavour to address a number of issues not addressed accurately including sensitization of mothers and healthcare providers on respectful maternity care, standardization of definitions of respectful maternity care tenets, quantifying the prevalence of disrespect and abuse since there is no enough documented data, examination of macro and micro level factors to disrespect and abuse, assess the impact of mistreatment on the health outcomes of women and their newborns and use mixed methods study design hence bridging the gap between observed measures and participant' self-reported experiences of mistreatment.

\section{ACKNOWLEDGEMENTS}

I would like to extend my appreciations to everyone who made this study feasible and achievement of my ideas and objectives a reality.

First and foremost, to my research partner Jacob Odhiambo Ngachra who spent sleepless nights searching literature. Secondly to my parents $\mathrm{Mr}$ Vincent Namusonge and Mrs Adah Namwaya for their constant support and encouragement, I can never thank you enough.

Thanks also to all other people who contributed in one way or another.

\section{REFERENCES}

Abbott, P., Burgess, K., Wang, E., \& Kim, K. (2010). Analysis of dentists' participation in continuing professional development courses from 2001-2006. The open dentistry journal, 4, 179.

Abuya, T., Ndwiga, C., Ritter, J., Kanya, L., Bellows, B., Binkin, N., \& Warren, C. E. (2015). The effect of a multi-component intervention on disrespect and abuse during childbirth in Kenya. BMC pregnancy and childbirth, 15(1), 1-14.

Afulani, P. A., Kirumbi, L., \& Lyndon, A. (2017). What makes or mars the facility-based childbirth experience: thematic analysis of women's childbirth experiences in western Kenya. Reproductive health, 14(1), 1-13.

Austad, K., Chary, A., Martinez, B., Juarez, M., Martin, Y. J., Ixen, E. C., \& Rohloff, P. (2017). Obstetric care navigation: a new approach to promote respectful maternity care and overcome barriers to safe motherhood. Reproductive health, 14(1), 1-8.

Austin, A., Langer, A., Salam, R. A., Lassi, Z. S., Das, J. K., \& Bhutta, Z. A. (2014). Approaches to improve the quality of maternal and newborn health care: an overview of the evidence. Reproductive health, 11(2), 1-9.

54 | This work is licensed under a Creative Commons Attribution 4.0 International License. 
Balde, M. D., Bangoura, A., Sall, O., Balde, H., Niakate, A. S., Vogel, J. P., \& Bohren, M. A. (2017). A qualitative study of women's and health providers' attitudes and acceptability of mistreatment during childbirth in health facilities in Guinea. Reproductive health, 14(1), 1-13.

Bartlett, R. (2015). Respectful maternity care is everyone's responsibility. Retrieved from IntraHealth International, https://www.intraheal th.org/vital/respectful- maternity- care- everyon e's-responsibility

Behruzi, R., Hatem, M., Fraser, W., Goulet, L., Ii, M., \& Misago, C. (2010). Facilitators and barriers in the humanization of childbirth practice in Japan. BMC pregnancy and childbirth, 10(1), 1-18.

Bingham, D., \& Main, E. K. (2010). Effective implementation strategies and tactics for leading change on maternity units. The Journal of perinatal \& neonatal nursing, 24(1), 32-42.

Bohren, M. A., Hunter, E. C., Munthe-Kaas, H. M., Souza, J. P., Vogel, J. P., \& Gülmezoglu, A. M. (2014). Facilitators and barriers to facility-based delivery in low-and middle-income countries: a qualitative evidence synthesis. Reproductive health, 11(1), 1-17.

Bohren, M. A., Titiloye, M. A., Kyaddondo, D., Hunter, E. C., Oladapo, O. T., Tunçalp, Ö., \& Mugerwa, K. (2017). Defining quality of care during childbirth from the perspectives of Nigerian and Ugandan women: A qualitative study. International Journal of Gynecology \& Obstetrics, 139, 4-16.

Bohren, M. A., Vogel, J. P., Hunter, E. C., Lutsiv, O., Makh, S. K., Souza, J. P., \& Gülmezoglu, A. M. (2015). The mistreatment of women during childbirth in health facilities globally: a mixedmethods systematic review. PLoS medicine, 12( 6), e1001847.

Bowser, D., \& Hill, K. (2010). Exploring evidence for disrespect and abuse in facility-based childbirth: Report of a landscape analysis. USAID-TRAction Project. University Research Cooperation, LLC, and Harvard School of Public Health.
Chalmers, B. (1992). WHO appropriate technology for birth revisited. British journal of obstetrics and gynaecology, 99(9), 709-710.

Côrtes, C. T., Oliveira, S. M. J. V. D., Santos, R. C. S. D., Francisco, A. A., Riesco, M. L. G., \& Shimoda, G. T. (2018). Implementation of evidence-based practices in normal delivery care. Revista latino-americana de enfermagem, 26, e2988.

Curl, M., \& Lothian, J. A. (2013). Evidence-based maternity care: can new dogs learn old tricks? The Journal of perinatal education, 22(4), 234240.

Das, M. K., Arora, N. K., Dalpath, S., Kumar, S., Qazi, S. A., \& Bahl, R. (2018). Improving quality of care for perinatal and newborn care at district and subdistrict hospitals in Haryana, India: Implementation research protocol. Journal of advanced nursing, 74(12), 2904-2911.

Dickson, K. E., Kinney, M. V., Moxon, S. G., Ashton, J., Zaka, N., Simen-Kapeu, A., ... \& Lawn, J. E. (2015). Scaling up quality care for mothers and newborns around the time of birth: an overview of methods and analyses of intervention- specific bottlenecks and solutions. BMC pregnancy and childbirth, 15(2), 1-19.

Esamai, F., Nangami, M., Tabu, J., Mwangi, A., Ayuku, D., \& Were, E. (2017). A system approach to improving maternal and child health care delivery in Kenya: innovations at the community and primary care facilities (a protocol). Reproductive health, 14(1), 1-18.

Furuta, M., Sandall, J., \& Bick, D. (2014). Women's perceptions and experiences of severe maternal morbidity-A synthesis of qualitative studies using a meta- ethnographic approach. Midwifer $y, 30(2), 158-169$.

Gabrysch, S., Civitelli, G., Edmond, K. M., Mathai, M., Ali, M., Bhutta, Z. A., \& Campbell, O. M. (2012). New signal functions to measure the ability of health facilities to provide routine and emergency newborn care. PLoS Med,9(11), e1001340.

55 | This work is licensed under a Creative Commons Attribution 4.0 International License. 
Gagliard, A. R., Légaré, F., Brouwers, M. C., Webster, F., Wiljer, D., Badley, E., \& Straus, S. (2011). Protocol: developing a conceptual framework of patient mediated knowledge translation, systematic review using a realist approach. Implementation Science, 6(1), 1-5.

George-Carey, R. (2018). Landmark Ruling in Kenya: A Victory for Respectful Maternity care. Boston, MA: Maternal Health Task Force, Harvard Chan School Center of Excellence in Maternal and Child Health

Haddou, L. (2014). Maternal mortality down $45 \%$ globally, but 33 women an hour are still dying. Retrieved from The Guardian, https://www.theguardian.com/news/datablog/20 14/may/07/maternal-mortality-rate-drops-halfreport-who-childbirth-pregnancy.

Hastings, M. B. (2015). Pulling back the curtain on disrespect and abuse: the movement to ensure respectful maternity care. Washington, DC: White Ribbon Alliance.

Hulton, L.A., Matthews, Z., \& Stones, R.W. (2000). A Framework for the Evaluation of Quality of Care in Maternity Services. University of Southampton, Southampton, United Kingdom.

Iyengar, K., Jain, M., Thomas, S., Dashora, K., Liu, W., Saini, P., \& Iyengar, S. (2014). Adherence to evidence-based care practices for childbirth before and after a quality improvement intervention in health facilities of Rajasthan, India. BMC pregnancy and childbirth, 14(1), 112.

Kenya National Bureau of Statistics. (2015). Kenya Demographic and Health Survey 2014. Kenya National Bureau of Statistics.

Kruk, M. E., Kujawski, S., Mbaruku, G., Ramsey, K., Moyo, W., \& Freedman, L. P. (2018). Disrespectful and abusive treatment during facility delivery in Tanzania: a facility and community survey. Health Policy and Planning, 33(1), e26-e33.

Ministry of Public Health and Sanitation \& Ministry of Medical Services. (2010). National Road Map for Accelerating the Attainment of the MDGs
Related to Maternal and Newborn Health, Kenya. Ministry of Public Health and Sanitation $\&$ Ministry of Medical Services.

Mirkuzie, A. H., Sisay, M. M., Reta, A. T., \& Bedane, M. M. (2014). Current evidence on basic emergency obstetric and newborn care services in Addis Ababa, Ethiopia; a cross sectional study. BMC pregnancy and childbirth, 14(1), 1-8.

Mselle, L. T., Kohi, T. W., \& Dol, J. (2018). Barriers and facilitators to humanizing birth care in Tanzania: findings from semi-structured interviews with midwives and obstetricians. Reproductive health, 15(1), 1-10.

Mwaniki, M. K., Vaid, S., Chome, I. M., Amolo, D., \& Tawfik, Y. (2014). Improving service uptake and quality of care of integrated maternal health services: the Kenya Kwale District improvement collaborative. BMC health services research, 14(1), 1-9.

Nababan, H. Y., Islam, R., Mostari, S., Tariqujjaman, M., Sarker, M., Islam, M. T., \& Moucheraud, C. (2017). Improving quality of care for maternal and newborn health: a pre-post evaluation of the Safe Childbirth Checklist at a hospital in Bangladesh. BMC pregnancy and childbirth, 17(1), 1-10.

Namusonge, L. N., Kabue, P. N., \& Sharma, R. R. (2016). Women's persistent utilisation of unskilled birth attendants in Kakamega County, Kenya. East African Medical Journal, 93(12), 691-711.

Ndwiga, C. (2017). Advancing respectful maternity care in Kenya: a comprehensive approach. Retrieved from Population Council, https://www.popcouncil.org/news/advancingrespectful-maternity-care-in-kenya-acomprehensive-approach.

Ndwiga, C., Warren, C. E., Ritter, J., Sripad, P., \& Abuya, T. (2017). Exploring provider perspectives on respectful maternity care in Kenya: "work with what you have". Reproductive health, 14(1), 1-13.

$$
56 \text { | This work is licensed under a Creative Commons Attribution 4.0 International License. }
$$


Nursing Council of Kenya. (2012). Continuing Professional Development Framework (CPD) for Nurses in Kenya. Nursing Council of Kenya.

Oliveira, A. S., Damasceno, A. K. C., Moraes, J. L., Moreira, K. A. P., Teles, L. M. R., \& Gomes, L. M. S. (2014). Technology used by companions in labor and childbirth: a descriptive study. Online Brazilian Journal of Nursing, 13(1), 36-45.

Oosthuizen, S. J., Bergh, A. M., Pattinson, R. C., \& Grimbeek, J. (2017). It does matter where you come from: mothers' experiences of childbirth in midwife obstetric units, Tshwane, South Africa. Reproductive health, 14(1), 1-11.

Ramsey, K., Ateva, E., Peca, E. (2016). From Anecdote to Action: catalyzing the Respectful Maternity Care movement.

Sando, D., Ratcliffe, H., McDonald, K., Spiegelman, D., Lyatuu, G., Mwanyika-Sando, M., \& Langer, A. (2016). The prevalence of disrespect and abuse during facility-based childbirth in urban Tanzania. BMC pregnancy and childbirth, 16(1), 1-10.

Savage, V., \& Castro, A. (2017). Measuring mistreatment of women during childbirth: a review of terminology and methodological approaches. Reproductive Health, 14(1), 1-27.

Silva, A. L. S., do Nascimento, E. R. \& Coelho, E. A. C. (2015). Nurses practices to promote dignity, participation and empowerment of women in natural birth. Esc. Anna Nery, 19(3), 424-431.

Siraj, A., Teka, W., \& Hebo, H. (2019). Prevalence of disrespect and abuse during facility based child birth and associated factors, Jimma University Medical Center, Southwest Ethiopia. BMC pregnancy and childbirth, 19(1), $1-9$.

Souza, J. P., Gülmezoglu, A. M., Vogel, J., Carroli, G., Lumbiganon, P., Qureshi, Z., \& Say, L. (2013). Moving beyond essential interventions for reduction of maternal mortality (the WHO Multicountry Survey on Maternal and Newborn
Health): a cross-sectional study. The Lancet, 381(9879), 1747-1755.

Stake, R. (2006). Multiple case study analysis. New York: The Guildford Press

Turab, A., Ariff, S., Habib, M. A., Ahmed, I., Hussain, M., Rashid, A., \& Bhutta, Z. A. (2013). Improved accessibility of emergency obstetrics and newborn care (EmONC) services for maternal and newborn health: a communitybased project. BMC pregnancy and childbirth, 13(1), 1-8.

Van den Broek, N. R., \& Graham, W. J. (2009). Quality of care for maternal and newborn health: the neglected agenda. BJOG: An International Journal of Obstetrics \& Gynaecology, 116, 1821.

White Ribbon Alliance. (2011). Respectful Maternity Care: the universal rights of childbearing Women. Washington, DC: White Ribbon Alliance for Safe Motherhood.

Wiegers, T. A. (2009). The quality of maternity care services as experienced by women in the Netherlands. BMC pregnancy and childbirth, 9(1), 1-11.

World Health Organization. (2014). Every Newborn: an action plan to end preventable deaths. World Health Organization.

World Health Organization. (2010). Quality of care; Patient safety. Report by the Secretariat. Fiftyfifth World Health Assembly, provisional agenda item 13.9. 2010.

World Health Organization. (2015a). Trends in maternal mortality: 1990-2015. Estimated from WHO, UNICEF, UNFPA, World Bank Group and the United Nations Population Division. World Health Organization.

World Health Organization. (2015b). WHO statement on prevention and elimination of disrespect and abuse during childbirth. Global Health Project.

World Health Organization. (2018). WHO recommendation on respectful maternity care

57 | This work is licensed under a Creative Commons Attribution 4.0 International License. 
East African Journal of Health and Science, Volume 3, Issue 1, 2021

Article DOI: https://doi.org/10.37284/eajhs.3.1.330

during labour and childbirth. Global Health Project.

World Health Statistics. (2016). Monitoring health for the SDGs, sustainable development goals. Geneva: World Health Organization.

58 | This work is licensed under a Creative Commons Attribution 4.0 International License. 